\title{
HIV modulates the expression of ligands important in triggering natural killer cell cytotoxic responses on infected primary T-cell blasts
}

\author{
Jeffrey Ward, ${ }^{1}$ Matthew Bonaparte, ${ }^{1}$ Jennifer Sacks, ${ }^{1}$ Jacqueline Guterman, ${ }^{1}$ Manuela Fogli, ${ }^{2}$ \\ Domenico Mavilio, ${ }^{2}$ and Edward Barker ${ }^{3}$ \\ ${ }^{1}$ Department of Microbiology and Immunology, State University of New York, Upstate Medical University, Syracuse, NY; ${ }^{2}$ Laboratory of Immunoregulation, \\ National Institute of Allergy and Infectious Diseases, National Institutes of Health, Bethesda, MD; ${ }^{3}$ Department of Immunology and Microbiology, Rush University \\ Medical Center, Chicago, IL
}

The ability of natural killer (NK) cells to kill virus-infected cells depends on the presence of ligands for activation receptors on the target cells. We found the presence of few, if any, NKp30 and NK46 ligands on T cell blasts infected with HIV, although NKp44 ligands were found on infected cells. HIV does induce the NKG2D ligands ULBP-1, -2 , and -3 . These ligands are involved in triggering NK cells to kill autologous HIV-infected cells, because interfering with the interaction between NKG2D, but not NKp46, on NK cells and its ligands on HIV-infected cells drastically reduced the lysis of infected cells. Interfering with the binding of the NK-cell coreceptors NTB-A and 2B4 to their ligands also decreased destruction by NK cells. The coreceptor ligands, NTB-A and CD48, were also found to be down- regulated during the course of HIV infection. Thus, ligands for NK-cell receptors are modulated during the course of HIV infection, which may greatly alter NK cells' ability to kill the infected cells. (Blood. 2007;110:1207-1214)

(C) 2007 by The American Society of Hematology

\section{Introduction}

NK cells are a distinct subset of cytotoxic lymphocytes that are an important first line of defense against several viruses that recognize and destroy infected cells without prior sensitization to viral antigens. The key role that NK cells play in protecting against certain viruses in vivo was initially highlighted in studies of mice depleted of NK cells and infected with mouse hepatitis virus, murine cytomegalovirus, and vaccinia virus. ${ }^{1}$

In humans, disseminated, life-threatening herpes virus infections occur in patients with NK-cell deficiencies. ${ }^{2}$ Moreover, low or dysfunctional NK-cell activity is associated with frequent herpes virus infections. ${ }^{3,4}$ The importance of NK-cell responses in controlling viruses is underscored by the observation that many viruses (eg, herpes, adeno-, papilloma, and hepatitis viruses) have developed mechanisms to thwart NK-cell responses. ${ }^{5}$ At this time, little is known about how HIV triggers NK cells to kill infected cells and whether the virus has developed mechanisms to prevent NK-cell-mediated clearance.

Unlike virus-infected cells, normal cells are protected from NK-cell destruction because major histocompatibility complex class I molecules (MHC-I) interact with inhibitory receptors (iNKR) present on NK cells. ${ }^{6-8}$ However, when viruses downmodulate MHC-I on infected cells to avoid virus-specific CTL recognition, infected cells can be destroyed by NK cells because infected cells lack the ligands that bind the NK-cell iNKR.,

Three major families of the iNKR receptors that interact with different MHC-I on target cells modulate NK cells. The first family of iNKR receptors is designated killer immunoglobulin (Ig)-like receptors (KIR), whose members have various numbers of Ig domains and ligand specificities. ${ }^{10,11}$ KIR with 2 Ig domains
(KIR2DL1, -2, and -3, also known as CD158a and -b) bind to different alleles of human leukocyte antigen C (HLA-C) molecules. ${ }^{7}$ KIR with 3 Ig domains (KIR3DL1) recognize different alleles of HLA-B and HLA-A (ie, HLA-Bw4 serotypes) molecules, ${ }^{12}$ and a homodimer of KIR3DL2 binds specific haplotypes of HLA-A molecules. ${ }^{13}$ The second family of iNKR receptors on NK cells is a lectin-like heterodimer consisting of CD94 associated with NKG2A/B, a molecule that binds to HLA-E, a nonclassic MHC-I. HLA-E presents leader peptides of HLA-A, -B, and -C on the cell surface. ${ }^{14,15}$ The last family of iNKR receptors, designated Ig-like transcripts-2, recognizes multiple HLA types. ${ }^{16}$ Even though the iNKR receptors differ in specificity, they all contain immunoreceptor tyrosine-based inhibitory motifs (ITIMs) in their cytoplasmic regions. ${ }^{16-18}$ The ITIMs function to recruit and lead to the activation of inhibitory phosphatases, such as Src-homology domain-containing tyrosine phosphatases (SHP)-1 and SHP-2. ${ }^{19}$

HIV-infected cells down-modulate MHC-I, ${ }^{20}$ and NK cells kill cells with low levels of MHC-I. ${ }^{9}$ Thus, it follows that HIV-infected cells should be susceptible to NK-cell-mediated killing. However, we have demonstrated that peripheral blood NK cells cannot kill HIV-infected, autologous T-cell blasts efficiently despite downmodulation of MHC-I. ${ }^{21}$ Cohen et $\mathrm{al}^{22}$ showed that selective down-regulation of MHC-I by HIV renders the infected cells resistant to NK-cell killing, and, because the HLA-C and -E remaining on the cell surface trigger iNKR, protection of the infected cells results. $\mathrm{We}^{20}$ and others ${ }^{23-26}$ have observed that, remarkably, HLA-C and -E iNKR are only expressed on a subset of NK cells. Therefore, we reasoned that HLA-C and $-\mathrm{E}$ prevent
Submitted June 12, 2006; accepted May 11, 2007. Prepublished online as Blood First Edition Paper, May 22, 2007; DOI 10.1182/blood-2006-06-028175.

The online version of this article contains a data supplement.

The publication costs of this article were defrayed in part by page charge payment. Therefore, and solely to indicate this fact, this article is hereby marked "advertisement" in accordance with 18 USC section 1734. 
some, but not all, NK cells from destroying HIV-infected cells. In support of this idea, we demonstrated that NK cells lacking HLA-C and $-\mathrm{E}$ iNKR kill autologous HIV-infected T cells. ${ }^{20}$ Because HIV-infected CD4-positive $\left(\mathrm{CD}^{+}{ }^{+}\right) \mathrm{T}$ cells are killed by NK cells regardless of HLA-C and -E presence, HLA-C and -E may not be solely responsible for restraining the response.

Lack of, or impaired expression of, MHC-I is not sufficient to account for killing by NK cells. ${ }^{27}$ Engagement of activating receptors on infected cells is required to trigger NK cells to kill. A variety of surface receptors can induce NK-cell activation. The Ig-like domain containing natural cytotoxicity receptors (NCR) NKp30, NKp44, and NKp46, and the C-type lectin NKG2D receptor, are described as the major NK-cell-activating receptors. ${ }^{28}$ It is thought that triggering NK-cell activation through NKG2D and the NCR can be regulated after engagement of iNKR, ${ }^{29-32}$ although the strength of the activating signal may overcome the effects of iNKR engagement. ${ }^{27}$

The cellular ligands for human NCR are not known at this time; however, influenza hemagglutinin has been identified as a viral ligand for the NKp46 receptor. ${ }^{33}$ In contrast, human NKG2D ligands consist of 2 classes of MHC-I like molecules: human cytomegalovirus UL-16 binding proteins (ULBP) and MHC-I related chain (MIC). These molecules are expressed only on "stressed" cells upon DNA damage and replication inhibitioninduced DNA repair pathways, ${ }^{34}$ and thus, signal NK-cellmediated destruction of the stress-ligand bearing cells.

In addition to the NCR and NKG2D, another set of NKcell-activating receptors has been identified that are categorized as coreceptors because of their ability to amplify NK-cell cytotoxicity when triggered along with a major activating receptor. ${ }^{35}$ Both the CD244/2B4 receptor and the NK-T-B cell antigen (NTB-A) are members of the $\mathrm{CD} 2$ subfamily of receptors, and their ligands have been identified as CD48 $8^{36}$ and NTB-A itself, respectively. ${ }^{37,38}$ Previous studies have shown that certain lymphoblastic leukemias are able to down-regulate the expression of CD48 and NTB-A, thereby escaping destruction by NK cells. ${ }^{39}$

Although HIV-infected cells can be killed by NK cells lacking iNKR to HLA-C and -E, it remains to be determined what triggers the NK cells to kill HIV-infected cells. Here we determined whether the ligands for NCRs and NKG2D, as well as the ligands for the NK-cell coreceptors, NTB-A and 2B4, on HIV-infected cells are important in triggering NK cells to kill the infected cells. In addition, we determined whether HIV modulates these molecules on infected cells.

\section{Materials and methods}

\section{HIV infection of CD4 ${ }^{+} \mathrm{T}$ cells}

Peripheral blood was obtained from healthy HIV seronegative donors after informed consent was obtained in accordance with the Declaration of Helsinki and the policies of the Institutional Review Board for the protection of human subjects at SUNY Upstate Medical University, Syracuse, NY, and Rush University Medical Center, Chicago, IL. CD4 ${ }^{+}$T cells were obtained from peripheral blood mononuclear cells and stimulated with phytohemagglutinin as described previously. ${ }^{21} \mathrm{CD} 4^{+}$T-cell blasts were infected with $10^{3} 50 \%$ tissue culture infective dose $\left(\mathrm{TCID}_{50}\right.$ ) (Figure 7 only) or $10^{4} \mathrm{TCID}_{50}$ of either of the R5 strains, HIV-1 ${ }_{\mathrm{SF} 162}$ or HIV- $1_{\mathrm{SF} 128 \mathrm{~A}}$, by spin-inoculation as described previously ${ }^{40}$ or spun with medium as an uninfected control. Five to 7 days after infection, cells were used in cytotoxicity assays or prepared for flow cytometric analysis.

\section{Antibodies}

The mouse antihuman CD4, CD48, and MICA/B antibodies were purchased from BD Biosciences (La Jolla, CA), and the anti-NTB-A antibody
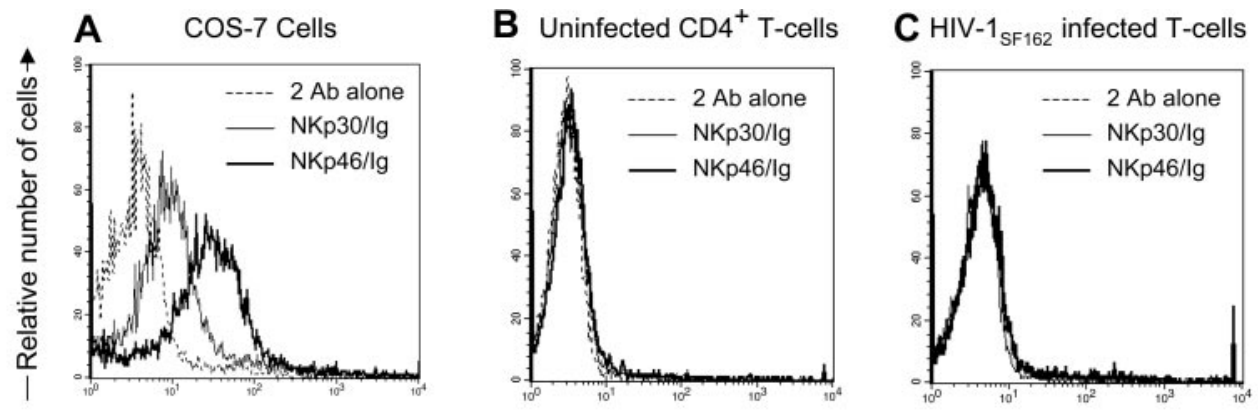

\section{Fluorescent intensity}
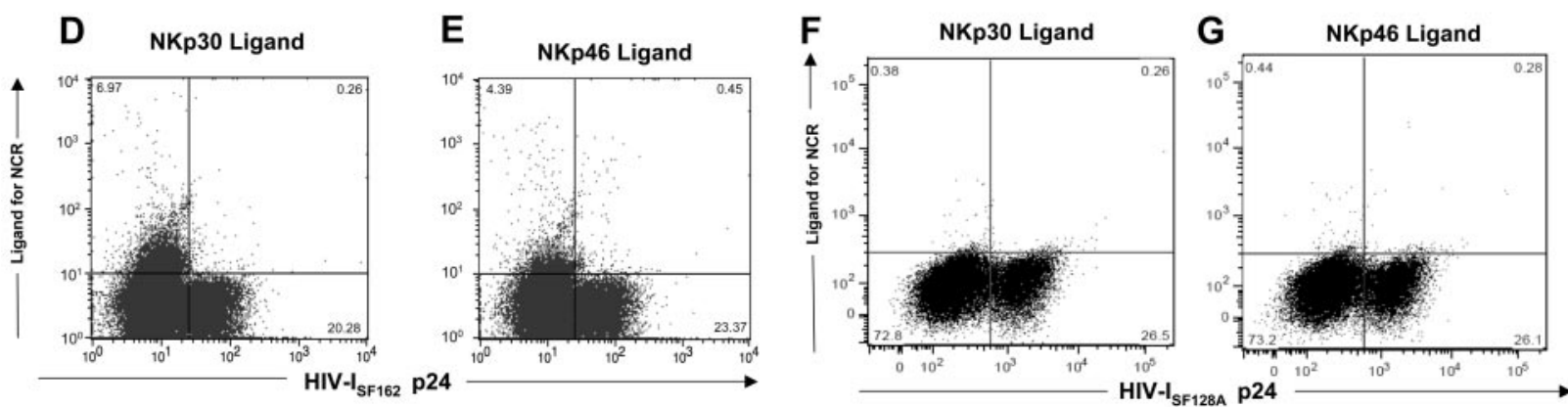

Figure 1. Expression of NKp30 or NKp46 ligands on HIV-infected primary T cells. (A) The COS-7 cell line was stained with either soluble NKp30-Fc or NKp46-Fc fusion proteins followed with goat antihuman secondary reagent or the secondary reagent alone. (B) Uninfected and (C) $\mathrm{HIV-1}$ SF162-infected CD4 ${ }^{+} \mathrm{T}_{\text {cells }}$ were stained with soluble NCR as in panel A, fixed and permeabilized, and stained with mouse anti-HIV-1 p24 monoclonal antibodies and rabbit antimouse secondary reagent. Histograms were gated on $10^{4}$ viable (panels A,B) or $10^{4} \mathrm{HIV}-1 \mathrm{p} 24 \mathrm{Ag}^{+}$cells (panel C). Dot plots shown are of $3 \times 10^{4}$ cells in the viable gate from cultures of T-cell blasts infected with HIV-1 $\mathrm{SF} 162$ (panels $\mathrm{D}, \mathrm{E}$ ) and $\mathrm{HIV}-1_{\mathrm{SF} 128 \mathrm{~A}}$ (panels $\mathrm{F}, \mathrm{G}$ ), and the numbers represent the percentage of viable cells within each quadrant. Data are representative of 3 separate studies. 
used for surface staining was purchased from Biolegend (San Diego, CA). The soluble fusion proteins of NKp30, NKp44, NKp46, and NKG2D receptors with the $\mathrm{Fc}$ portion of human IgG and mouse antihuman ULBP-1, -2 , or -3 antibodies were obtained from R\&D Systems (Minneapolis, MN). The following monoclonal antibodies were a kind gift from Dr A. Moretta (University of Genoa, Genoa, Italy): MA127 and ON56 (IgG1 and IgG2b isotypes, respectively, anti-NTB-A antibody), PP35 and CO54 (IgG1 and IgM isotypes, anti-2B4 antibody), and BAT221 (IgG1, anti-NKG2D antibody). Allophycocyanin (APC) and fluorescein isothiocyanate (FITC)conjugated goat antihuman IgG Fc-specific antibodies were obtained from Jackson ImmunoResearch Laboratories (West Grove, PA). FITC-conjugated rabbit antimouse $\mathrm{IgG} \mathrm{Fc}$-specific antibodies were from Dako Colorado (Fort Collins, CO). The 2 anti-HIV-1 p24 monoclonal antibodies used for intracellular flow cytometry staining were obtained from the National Institutes of Health AIDS Research and Reference Reagent Program (Germantown, MD). The human monoclonal antibody clone 71-31 was deposited by Dr S. Zolla-Pazner, ${ }^{41}$ and the mouse monoclonal antibody clone AG3.0 by Dr J. Allan. ${ }^{42}$ The mouse anti-HIV-1 p24 monoclonal antibody, KC57, was purchased from Beckman Coulter (Fullerton, CA). See Figure S1 (available on the Blood website; see the Supplemental Materials link at the top of the online article) for details of the staining patterns of various clones of anti-HIV-1 p24 antibodies.

\section{Staining and analysis of uninfected and HIV-infected T-cell blasts for ligands of NK-cell activation receptors and coreceptors}

Uninfected and HIV-infected CD4 ${ }^{+}$T-cell blasts were washed 3 times with $\mathrm{Ca}^{2+} / \mathrm{Mg}^{2+}$-free phosphate-buffered saline with $2 \%$ fetal bovine serum and $0.1 \% \mathrm{NaN}_{3}$ (flow buffer). After the final wash, $10^{6}$ of either uninfected or HIV-infected CD4 ${ }^{+}$T-cell blasts in flow buffer at a concentration of $10^{7} / \mathrm{mL}$ were stained with titered concentrations of soluble fusion proteins or monoclonal antibodies for 20 minutes at $4^{\circ} \mathrm{C}$. After washing samples twice with flow buffer, the cells were stained with the appropriate fluorochromeconjugated secondary reagents, washed twice in flow buffer and fixed and permeabilized as described previously. ${ }^{21}$ After perm-fixing, cells were stained with either human or mouse anti-HIV-1 p24 antibody as described previously. ${ }^{20,21}$ Viable uninfected and/or HIV-p24 Ag-positive $\left(\mathrm{Ag}^{+}\right)$cells were acquired using a LSR II flow cytometer (BD Biosciences, San Jose, CA). Acquired samples were analyzed using the Cellquest program (BD Biosciences) or FlowJo (Treestar, Ashland, OR).

\section{Cytotoxicity assay}

Target cells for ${ }^{51} \mathrm{Cr}$ release assays were prepared by depletion of $\mathrm{CD} 4^{+} \mathrm{T}$ cells from HIV-infected cells as described previously. ${ }^{20}$ Purity of the infected cells after depletion was less than $5 \% \mathrm{CD}^{+}$and $\mathrm{CD} 4$-negative $\left(\mathrm{CD}^{-}{ }^{-}\right.$) cells were more than $95 \%$ HIV-1 p24 Ag + as determined by flow cytometric analysis (see Figure S2). NK cells were obtained from the same donors who provided $\mathrm{CD} 4^{+} \mathrm{T}$ cells for our studies using immunomagnetic bead isolation with anti-CD56 antibody coupled to magnetic beads (Stem Cell Technologies, Vancouver, BC, Canada) as described previously. ${ }^{20}$ CD56-positive $\left(\mathrm{CD} 6^{+}\right)$cells were depleted of CD3-positive $\left(\mathrm{CD}^{+}\right)$cells using anti-CD3 antibody coupled to magnetic beads according to the manufacturer's instructions (Dynal, Brown Deer, WI) and rested overnight before use in cytotoxicity assays. NK cells were not treated with cytokines at any time. Saturating concentrations $(10 \mu \mathrm{g} / \mathrm{mL})$ of specific monoclonal antibodies blocking NK-cell receptors were added for 30 minutes at $4^{\circ} \mathrm{C}$ before and at $37^{\circ} \mathrm{C}$ during the cytotoxicity assays. Determination of NK-cell killing of HIV-infected cells was done using the ${ }^{51} \mathrm{Cr}$ release assay as described previously. ${ }^{20}$

\section{Results}

Determining the expression of ligands for natural cytotoxicity receptors on HIV-infected cells

Because triggering natural cytotoxicity receptors (NCR) on NK cells leads to destruction of NCR ligand-expressing target cells, ${ }^{43,44}$ we wanted to determine whether HIV-infected $\mathrm{CD} 4^{+} \mathrm{T}$ cells expressed the NKp30 and NKp46 ligands. For this purpose, we stained the surface of primary $\mathrm{CD}^{+} \mathrm{T}$ cells infected with 2 different strains of HIV-1 and uninfected CD4 ${ }^{+} \mathrm{T}$ cells with soluble human NKp30-Ig and NKp46-Ig fusion proteins. To detect cells possessing virus, we stained for intracellular HIV-1 p24 Ag. As a positive control for detecting NCR ligands, we used NKp30positive $\left(\mathrm{NKp} 0^{+}\right)$and $\mathrm{NKp} 46$-positive $\left(\mathrm{NKp} 46^{+}\right)$cell lines as control. ${ }^{45}$ Although NKp30 and NKp46 ligands are detected on the surface of the cell lines COS-7 (Figure 1A) and 293T (data not shown), they were absent on uninfected $\mathrm{CD}^{+}{ }^{+} \mathrm{T}$ cells (Figure 1B). Only a small percentage $(6.97 \%$ and $4.39 \%$, respectively) of HIV-1 $1_{\mathrm{SF} 162}$-infected cells expressed NKp30 and NKp46 ligand (Figure 1C-E). Cells infected with the HIV$1_{\mathrm{SF} 128 \mathrm{~A}}$ strain did not express the NKp30 and NKp46 ligands (Figure 1F,G).

Recent studies demonstrated that $\mathrm{CD}^{+} \mathrm{T}$ cells from subjects infected with HIV express the NKp44 ligand. ${ }^{46}$ In the present study, we wanted to determine whether HIV-infected $\mathrm{CD}^{+}{ }^{+} \mathrm{T}$ cells expressing
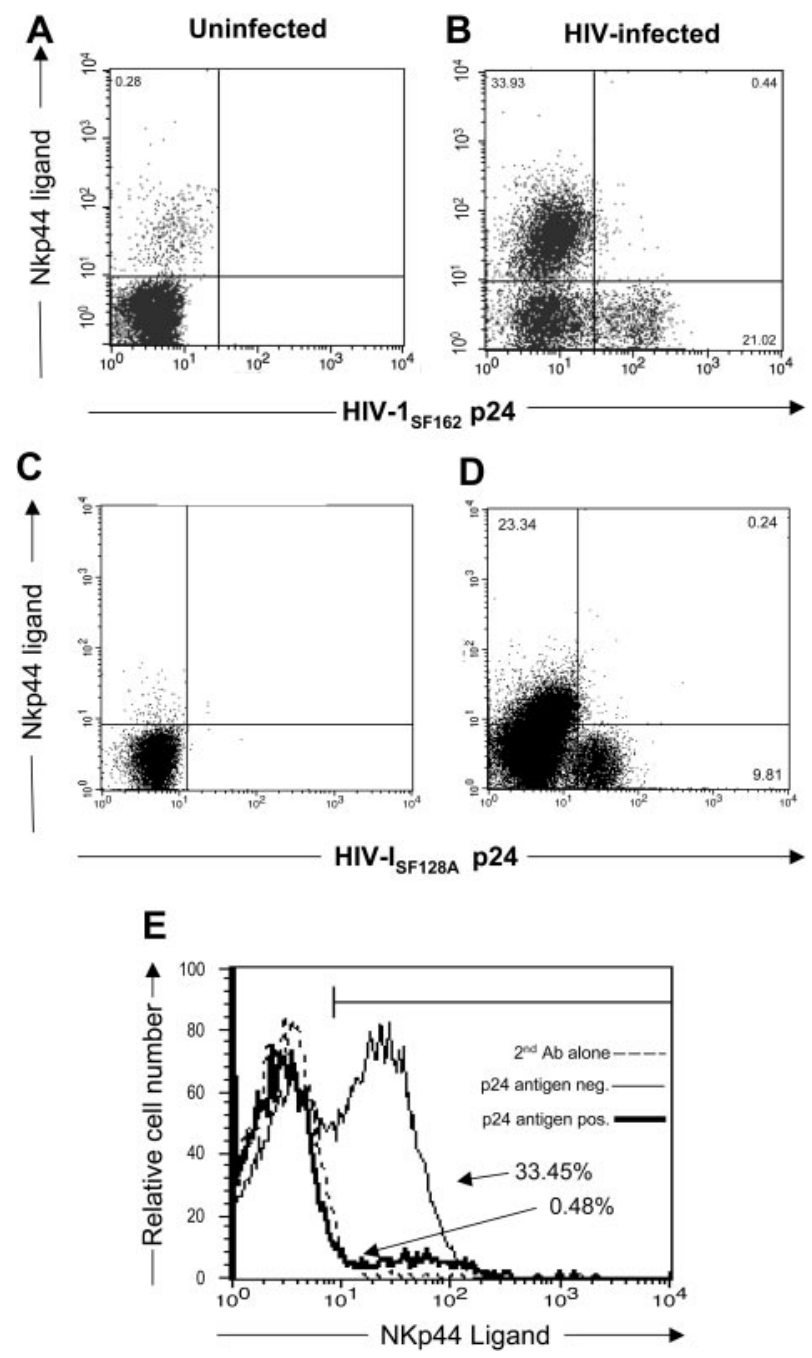

Figure 2. Expression of NKp44 ligands on HIV-infected primary $\mathrm{T}$ cells.

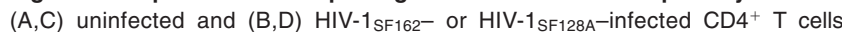
stained with soluble NKp44/lgG Fc fusion protein and goat antihuman secondary reagent followed by intracellular staining with mouse anti-HIV-1 p24 antibodies. Dot plots shown are of $3 \times 10^{4}$ cells in the viable gate from cultures of T-cell blasts infected with HIV, and the numbers represent the percentage of viable cells within each quadrant. (E) The percentage of HIV-1 p24 $\mathrm{Ag}^{+}(-)$and p24 $\mathrm{Ag}^{-}(\longrightarrow)$ infected cells that are NKp44 ligand-positive. Data are representative of 3 separate studies. 
virus possess the NKp44 ligand. To demonstrate the presence of NKp44 ligand, we stained HIV-infected and uninfected $\mathrm{CD} 4^{+} \mathrm{T}$ cells with an NKp44-Ig fusion protein. We also stained cells for intracellular HIV-1 p24 Ag. Figure 2A,C demonstrate limited expression of NKp44 ligand on uninfected $\mathrm{CD}^{+}{ }^{+} \mathrm{T}$ cells $\left(0.28 \%\right.$ of $\mathrm{CD}^{+}{ }^{+}$cells). However, when infected with $\mathrm{HIV}-1_{\mathrm{SF} 162}, \mathrm{CD} 4{ }^{+} \mathrm{T}$ cells express NKp44 ligands in $33.93 \%$ of the cells (Figure 2B,E). Infection with another HIV strain, HIV-1 $1_{\mathrm{SF} 128 \mathrm{~A}}$, also induced NKp44 ligand on the cells (23.34\% [Figure 2D]). These studies demonstrate that HIVinfected cells express little, if any, NKp30 and NKp46 ligands, although they did express NKp44 ligands.

\section{HIV-infected cells express the NKG2D ligands ULBP-1, -2, and -3}

$\mathrm{NKG} 2 \mathrm{D}$, an activation receptor on NK cells, is capable of triggering NK cells to kill target cells expressing its ligands. ${ }^{47}$ To determine whether HIV-infected cells express the ligands for NKG2D, we stained cells with soluble NKG2D-Ig fusion protein. We also stained cells for intracellular HIV-1 p24 Ag. As demonstrated in Figure 3A,E, NKG2D ligands are present on the infected cell surface. These same ligands are absent on uninfected $\mathrm{CD} 4^{+} \mathrm{T}$ cells (Figure 3B). NKG2D ligands are evident on the infected cells expressing detectable HIV-1 p24 antigen (Figure 3D,F), but few are found on the HIV-1 p24-negative population (Figure 3C). Thus, NKG2D ligands are induced on HIV-infected cells containing detectable HIV antigen.

Human NKG2D ligands consist of the MHC-I like molecules ULBP-1, -2, and -3 , as well as MIC-A and -B. ${ }^{48}$ We determined which ligands are present on HIV-infected cells. Antibodies directed to the various ULBP and MIC molecules were used to detect specific NKG2D ligands on infected cells. Figure 4A-C demonstrates the presence of all 3 ULBPs on HIV-1 $1_{\mathrm{SF} 162^{-}}$ infected cells. Neither MICA nor MICB is present on HIVinfected cells (Figure 4D-F), but can be seen on HeLa cells. Thus, HIV induces ULBP-1, -2 , and -3 but not MICA or MICB on infected $\mathrm{CD} 4^{+} \mathrm{T}$ cells.
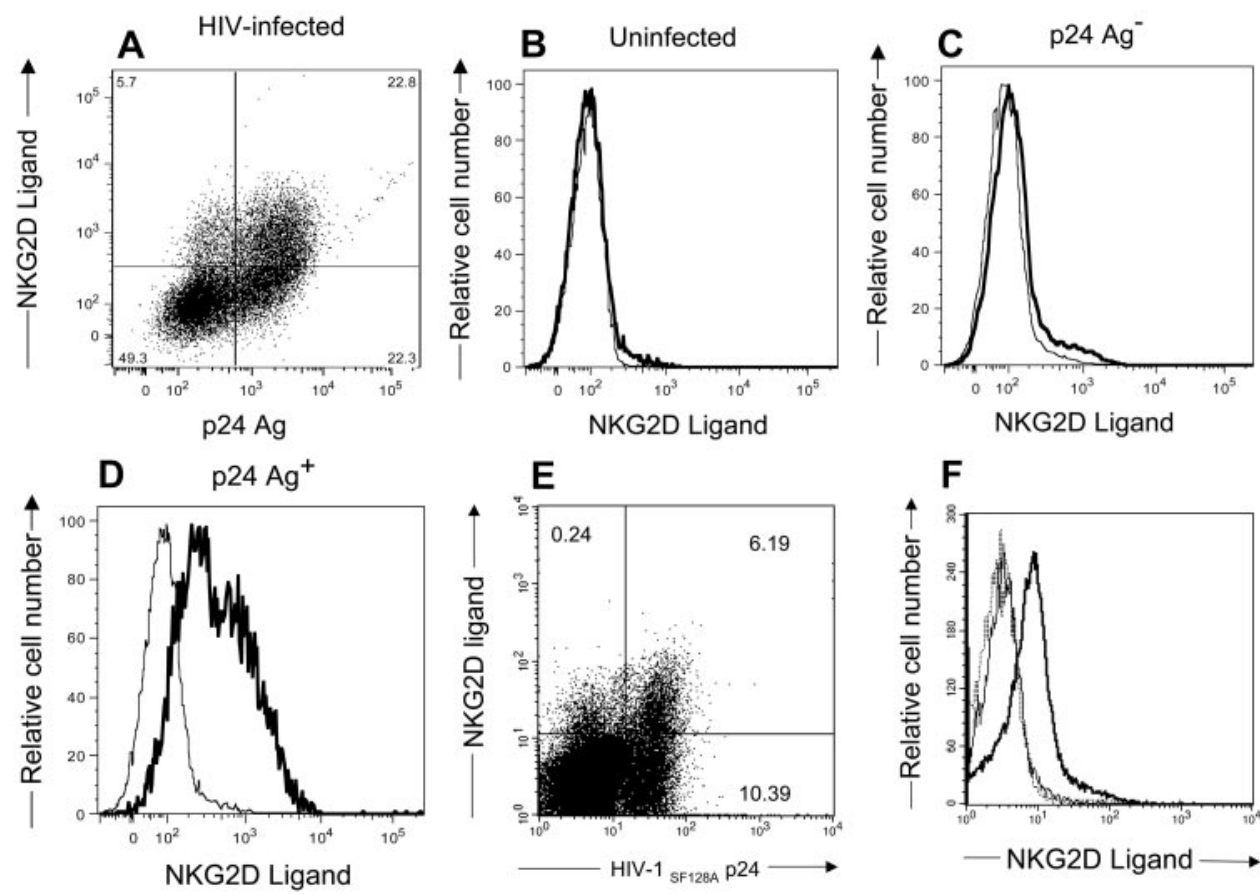

\section{Ligands for NKG2D trigger NK cells to kill HIV-infected cells}

Because HIV-infected cells express NKG2D ligands, we wanted to determine whether the ligands trigger NK cells to kill HIV-infected cells. For this purpose, we evaluated the ability of NK cells to destroy autologous HIV-infected cells (CD4-/HIV-1 p24 $\mathrm{Ag}^{+}$) in a 4-hour ${ }^{51} \mathrm{Cr}$ release assay in the presence of interfering antibodies to NKG2D. As shown in Figure 5, we were able to significantly decrease $(P<.05)$ the ability of NK cells to kill HIV-infected cells when NKG2D's ability to bind to its ligands was blocked. The specificity of this interaction is confirmed; blocking NK cells with an anti-NKp46 monoclonal antibody did not significantly reduce killing (Figure 5D), because NKp46 ligands are not expressed on HIV-infected cells (Figure 1C-E). Thus, NKG2D ligands present on HIV-infected cells are able to induce the cytotoxic response of NK cells.

\section{NTB-A and CD48 trigger the killing of autologous infected CD4 ${ }^{+}$ cells by NK cells and are down-modulated during the course of HIV-1 infection}

Both NTB-A and 2B4 serve as coreceptors during NK-cell activation and thus augment NK-cell killing when one of the major NK-cell receptors is also triggered. ${ }^{28} \mathrm{NTB}-\mathrm{A}$ is triggered through homophilic binding, whereas $2 \mathrm{~B} 4$ recognizes the GPI-linked protein $\mathrm{CD} 48$. Both of these ligands are present on $\mathrm{CD}^{+}$cells. Because we found NKG2D ligands on HIV-infected cells (Figures 3-4), we investigated the possibility that NTB-A and CD48 also serve as coactivating ligands on infected $\mathrm{CD}^{+}{ }^{+}$cells to trigger NK-cellmediated cytotoxicity. HIV-infected cells (CD4-/HIV-1 p24 $\mathrm{Ag}^{+}$) were used as targets in a 4-hour ${ }^{51} \mathrm{Cr}$ release assay using NK cells treated with either an isotype control antibody or masked with anti-NTB-A or anti-2B4 monoclonal antibodies as effectors. A significant $(P<.05)$ decrease in target cell destruction was detected after masking of the NK-cell coreceptors, indicating that NK-cell killing of autologous HIV-infected blasts depends on NTB-A and 2B4 (Figure 6).
Figure 3. CD4+ T-lymphocytes infected with HIV-1 express NKG2D ligands. CD4 ${ }^{+}$ $T$ cells $(B, F)$ uninfected or $(A, C, D)$ infected with HIV-1 SF162 $_{\text {or }}(\mathrm{E}, \mathrm{F})$ HIV-1 SF128A $_{\text {A }}$ were stained with NKG2D/lgG Fc fusion proteins and goat antihuman secondary reagent followed by staining with mouse anti-HIV-1 p24 antibodies. Numbers in the dot plots represent the percentage of viable cells within each quadrant. Histograms (panels B-D) show expression of NKG2D ligand on uninfected, HIV-1 p24 $\mathrm{Ag}^{-}$infected cells and HIV-1 p24 $\mathrm{Ag}^{+}$infected cells, respectively. (F) The histogram with the dotted line represents cells stained with secondary antibodies alone, the histogram with the thin line represents uninfected cells, and the histogram with the bold line represents HIV-1 p24 $\mathrm{Ag}^{+}$cells. These are representative of 5 separate experiments. 
Figure 4. CD4+ T-lymphocytes infected with HIV-1 express NKG2D ligands ULBP $-1,-2$, and -3 but not MICA or MICB. (Top panels) Uninfected and HIV$1_{S F 162}$-infected primary T-cell blasts were stained with antibodies directed to ULBP-1, -2 , and -3 , and a rabbit antimouse secondary reagent followed by intracellular staining with a monoclonal human anti-p24 antibody. (Bottom panels) The NKG2D ligands MICA and MICB were detected using specific antibodies on HIV-1 p24 $\mathrm{Ag}^{+}$infected cells and uninfected $\mathrm{CD}^{+}{ }^{+} \mathrm{T}$ cells. The HeLa cell line was used as a positive control for MICA and MICB expression. MFI = mean fluorescent intensity of antibody used for staining the cells. Data are representative of 3 separate studies.
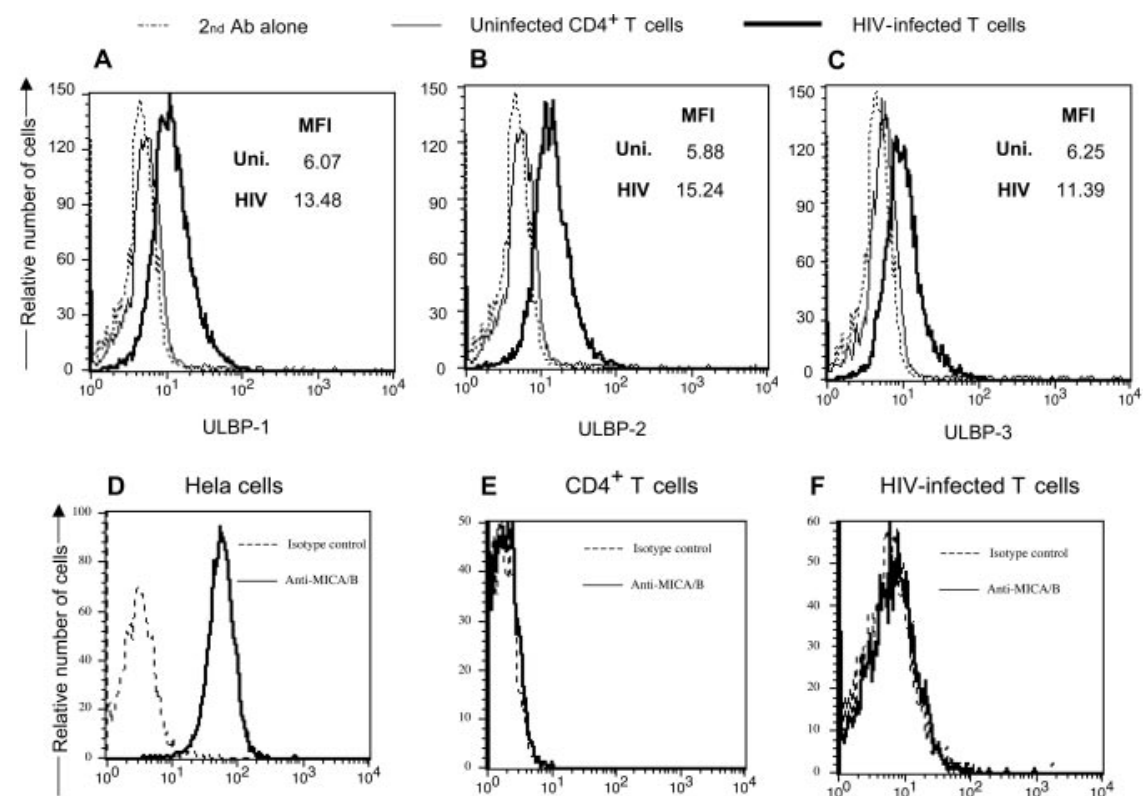

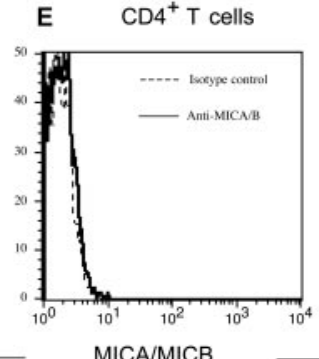

F HIV-infected T cells

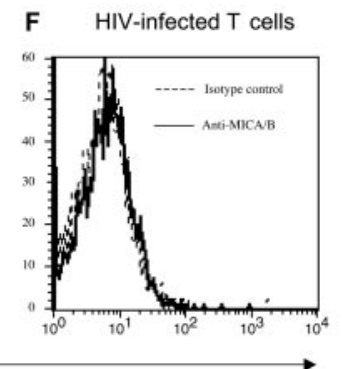

Because HIV is able to modulate MHC-I, CD4, and NKG2D ligand expression on infected target cells, we also investigated the possibility that the surface levels of NTB-A and CD48 were altered on virus-infected cells. During infection with 2 R5 strains, both CD48 (Figure 7A,C,D) and NTB-A (Figure 7B,E,F) are downmodulated from the cell surface.

\section{Discussion}

We demonstrated in this study that, on HIV-infected cells, NKG2D ligands are responsible for triggering NK cells to kill HIV-infected cells. The amount of killing of HIV-infected cells by autologous NK cells varies from donor to donor and ranges from less than $1 \%$ to as high as $30 \%$ at an E/T ratio of 10:1 in donors we have tested. Only studies that show a detectable amount of killing have been included here. It is noteworthy that although the majority of $\mathrm{CD}^{2} 6^{+}, \mathrm{CD} 3$-negative $\left(\mathrm{CD}^{-}\right)$NK cells are NKG2D-positive $\left(\mathrm{NKG}_{2} \mathrm{D}^{+}\right)$, there was no correlation between the level of NKcell-mediated killing of HIV-infected cells and the percentage of NK cells that were $\mathrm{NKG}_{2} \mathrm{D}^{+}$(see Figure S3). In contrast, the percentage of cells that is double-positive for NKG2D and the HLA-E binding inhibitory receptor, NKG2A, differs dramatically among the donors used in this study and corresponds to the level of killing observed. This is important because HLA-E molecules remain on the cell surface during HIV infection. ${ }^{20,22}$ However, it should be pointed out that other factors besides inhibitory or activating receptors may come into play with regard to the variability observed in NK killing of HIV-infected cells, because there are a wide range of responses even concerning NK-cell killing of MHC class I negative K562 target cells. ${ }^{20}$

The NKG2D ligands on HIV-infected cells that trigger NK cells are ULBP-1, -2, and -3, but not MICA or MICB (Figure 4). All 3 ULBPs are equally likely to stimulate NK cells to kill. ${ }^{49}$ We show that the ligands for the NTB-A and 2B4 coreceptors are responsible for inducing NK-cell killing. We also demonstrate that NCR ligands are not likely to trigger NK cells to destroy HIV-infected cells because few if any NCR ligands are expressed on the infected cell surface.

Ligands for the NCRs NKp30 and NKp46 are expressed on few, if any, uninfected or HIV-infected T cells (Figure 1). They are, however, expressed by tumor cell lines (Figure 1A), in
A

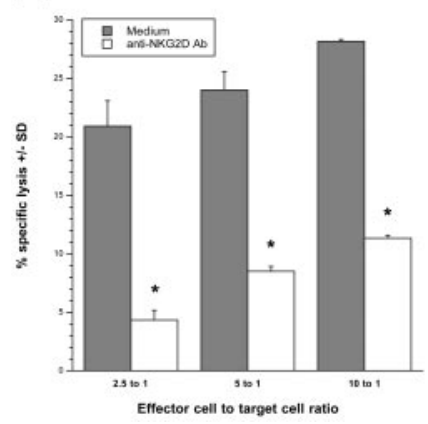

B

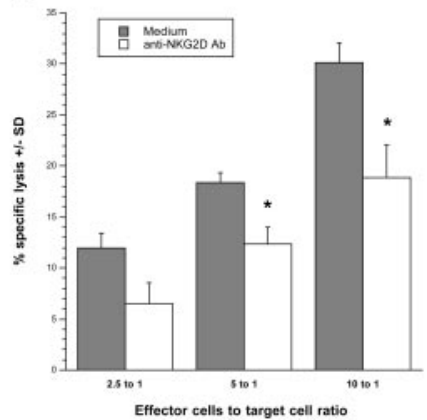

C

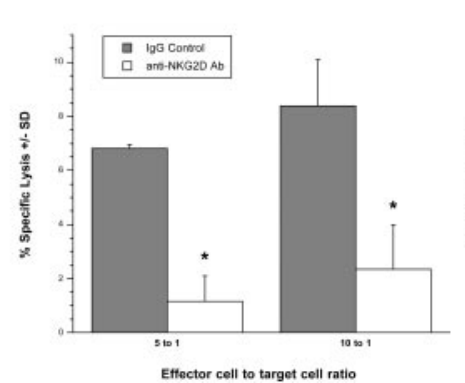

D

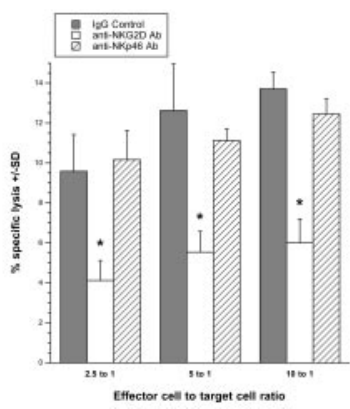

Figure 5. Interfering with the interaction between NKG2D and its ligands on HIV-infected T cells decreases NK-cell killing of the infected cells. (A,B,D) CD4-/HIV-1

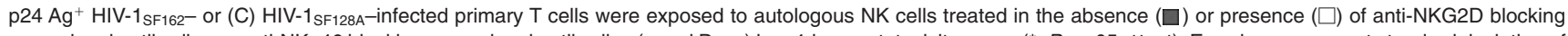
monoclonal antibodies or anti-NKp46 blocking monoclonal antibodies (panel D, ש $\boldsymbol{U}_{2}$ ) in a 4-hour cytotoxicity assay $\left({ }^{*}, P<.05 ; t\right.$ test). Error bars represent standard deviation of the mean. Panels $A, B$, and $D$ are 3 separate donors; panels $C$ and $D$ are from the same donor. 

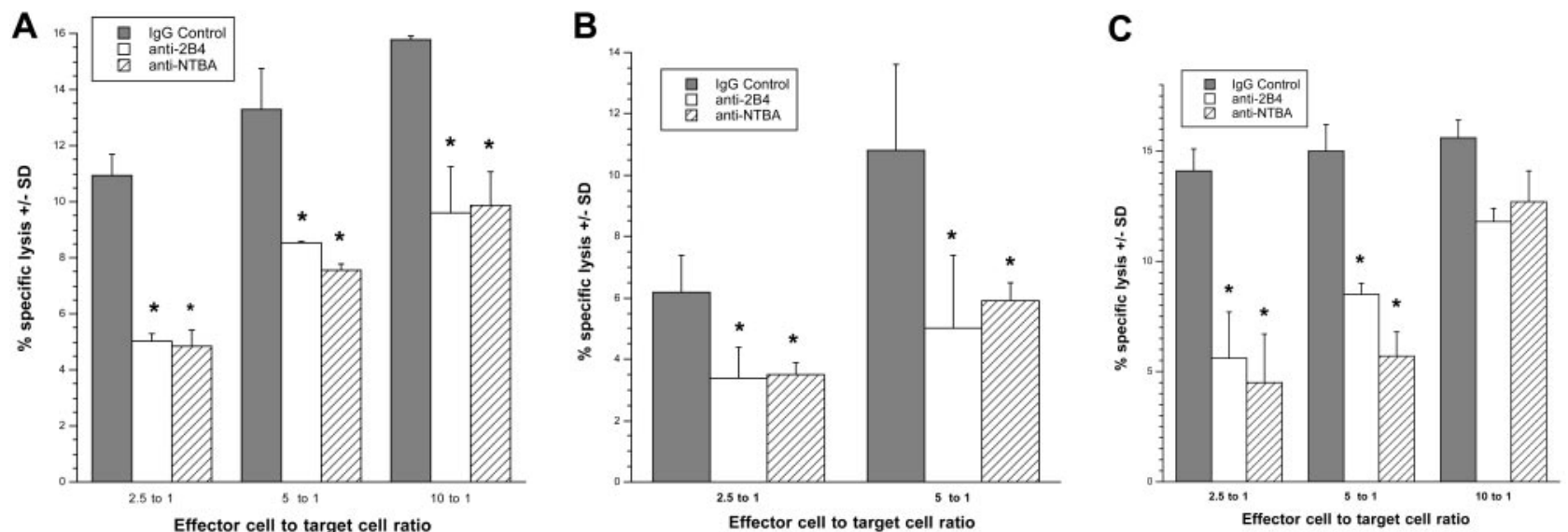

Figure 6. Preventing the interaction between NK-cell coreceptors and their ligands on HIV-infected cells decreases NK-cell killing of the targets. (A,B) CD4-/HIV-1 p24 Ag ${ }^{+}$HIV-1 SF128A-infected or (C) HIV-1 $_{\text {SF162 }}$-infected primary T cells were exposed to autologous NK cells treated in the absence (isotype control) or presence of anti-2B4 or NTB-A blocking monoclonal antibodies in a 4-hour cytotoxicity assay. Panels A through $\mathrm{C}$ represent 3 separate experiments involving 3 different donors ( ${ }^{*}, P<.05 ; t$ test). Error bars represent standard deviation of the mean.

agreement with results from previous studies. ${ }^{45}$ As reported previously, NKp44 ligands are expressed on cells when exposed to peptides found in HIV-1 gp41 or when exposed to soluble HIV-1 gp $160 .{ }^{46}$ In fact, a significant percentage of NKp44 ligand was seen on HIV-infected cells exposed to the virus. However, this up-regulation of ligand does not affect the cytotoxicity measured, because NKp44 is only expressed on cytokine-stimulated NK cells ${ }^{50,51}$ and is not found on the resting NK cells used in our assays.

Although NCR ligands are not found on HIV-1 p24 Ag ${ }^{+}$cells, the NKG2D ligands are present on HIV-infected cells. The level of NKG2D ligand expression is comparable with that found on previously reported cell lines. ${ }^{32}$ The expression of NKG2D ligands was mostly limited to HIV-1 p24 Ag-expressing HIV-infected cells. This is important with respect to the recognition of HIV-infected cells by NK cells, in that only HIV-infected cells down-modulate MHC-I molecules, which, in turn, inhibit NK-cell responses. It should be pointed out that not all infected cells express NKG2D ligands, and other NK-cell activation ligands may be involved in triggering NK-cell cytotoxicity. Moreover, blocking NK-cell kill- ing was not complete despite interfering with the interaction between NKG2D and its ligands with blocking antibody (Figure 5). Although both HLA-C and -E molecules have specific activation receptors on NK cells, we have ruled out the role of these activating receptors in triggering NK cells to kill HIV-infected cells in our previous studies. ${ }^{20}$ Thus, we endeavor to determine if novel activating ligands on HIV-infected cells trigger NK cells.

The specific NKG2D ligands expressed on HIV-1 p24 $\mathrm{Ag}^{+}$ HIV-infected cells were ULBP 1-3, but not MICA or MICB. This is in agreement with a recent study, which demonstrated that HIVinfected primary $\mathrm{CD}^{+}{ }^{+} \mathrm{T}$ cells express ULBP-1 and -2 but not MICA on the surface. ${ }^{52}$ Studies by Molinero et al ${ }^{53}$ demonstrated the expression of MICA on $63 \%-84 \%$ of $\mathrm{CD}^{+}{ }^{+} \mathrm{T}$ cells. We found no expression of MICA on $\mathrm{CD}^{+}{ }^{+} \mathrm{T}$ cells, despite the presence of the antigen on HeLa cells. We used 2 different clones of monoclonal anti-MICA antibody and did not detect this protein on the CD4 ${ }^{+}$ T-cell surface (data not shown). Molinero et $\mathrm{al}^{53}$ used polyclonal anti-MICA rabbit sera rather than monoclonal antibodies. High fluorescent background levels were detected in cells stained with normal rabbit sera (11\%-32\% positive) whereas less than $0.1 \%$ of
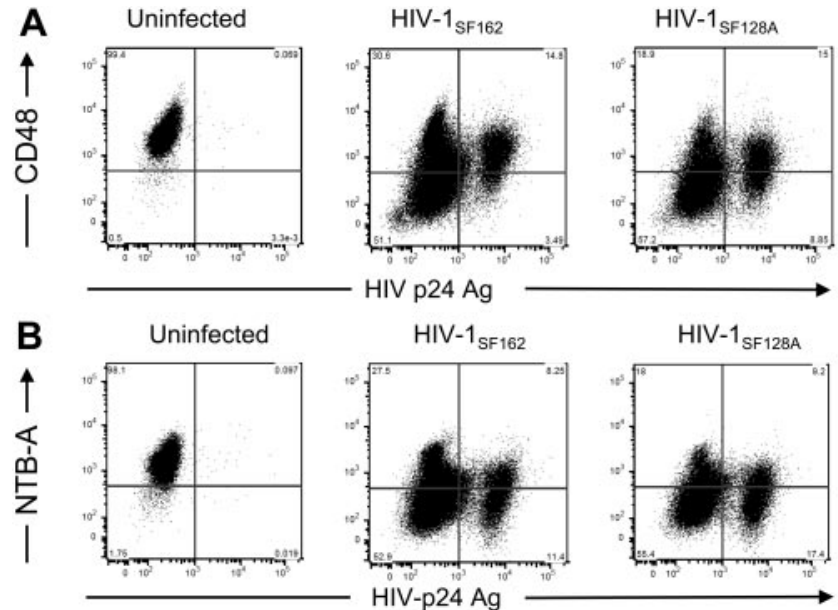
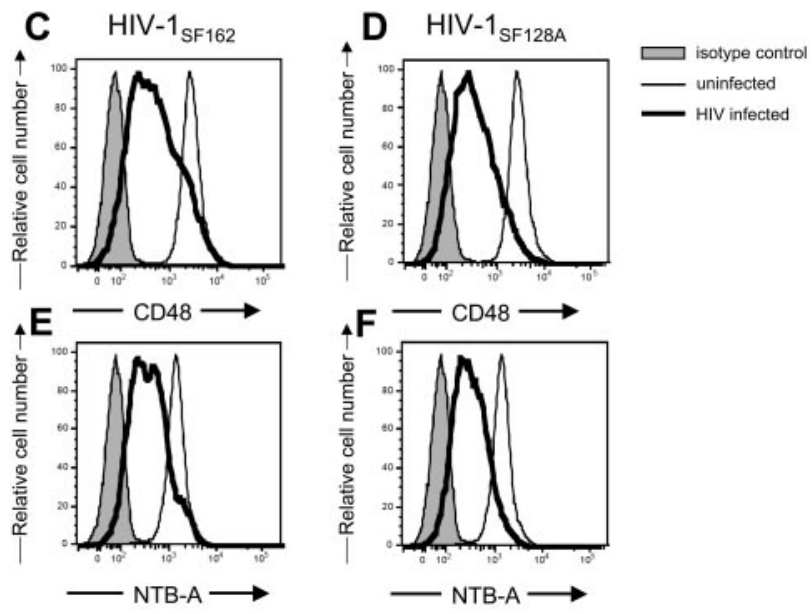

Figure 7. HIV-1 down-modulates NTB-A and CD48 on infected cells. Primary T-cell blasts either were left uninfected or were infected with $10^{3}$ TCID 50 HIV-1 SF162 or

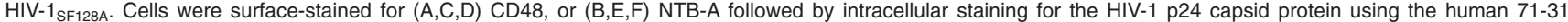
monoclonal antibody and goat antihuman secondary antibody. Data are representative of at least 3 different experiments with different donors. In panels $A$ and $B$, the numbers in the corners of the plot quadrants are the percentage of viable cells that express the specific markers. Histogram representation of all viable cells is shown in panels $C$ through $\mathrm{F}$ (匹 represents isotype control staining,___ represents surface staining of viable uninfected cells, and — represents surface staining of viable HIV-infected cells). 
PHA-stimulated $\mathrm{CD}^{+}$cells were positive using mouse isotype control antibodies in our study. Despite the high level of background staining with normal rabbit sera, the difference in antiMICA staining may be attributable to the absence of IL-2 in the medium of stimulated cells in the studies by Molinero et al. ${ }^{53}$ IL-2 (200 U/mL) was present during the stimulation and culture of $\mathrm{CD}^{+}{ }^{+} \mathrm{T}$ cells in our studies. Lack of IL-2 during stimulation may induce cell stress, thereby leading to induction of MICA expression.

We show here for the first time that NKG2D ligands on HIV-infected cells trigger $\mathrm{NK}$ cells to kill in the absence of antibodies and cytokine exposure. This is surprising because previous reports have indicated that NKG2D alone may not be able to trigger "resting" NK cells to kill cell lines and that coreceptors may be involved in triggering resting NK cells to degranulate.${ }^{54}$ For example, crosslinking NKG2D and 2B4 on unstimulated NK cells has been shown to increase $\mathrm{Ca}^{2+}$ flux and degranulation compared with either receptor alone. ${ }^{54} \mathrm{We}$ found that the ligands of the coreceptors $2 \mathrm{~B} 4$ and NTB-A are found on $\mathrm{CD} 4{ }^{+} \mathrm{T}$ cells. We did not observe the expression of CRACC or the DNAM ligand CD155 on $\mathrm{CD} 4^{+} \mathrm{T}$ cells (data not shown).

It is noteworthy that both NTB-A and CD48 are downmodulated on the surface of HIV-infected cells compared with uninfected control cells. The suboptimal triggering of $2 \mathrm{~B} 4$ and NTB-A together with NKG2D ligands may still be enough to trigger resting NK cells to kill HIV-infected targets, although it should be pointed out that killing may not be optimal if the coreceptor ligand expression was not at the levels found on uninfected cells. ${ }^{54}$ One way to evaluate this is to cross-link $2 \mathrm{~B} 4$ and NTB-A on NK cells when exposed to HIV-infected cells. The interactions of all 3 receptors for proper assessment of their respective contributions to a triggering signal would be desirable. However, we have been unable to purify a sufficiently large number of unstimulated NK cells and infected primary $\mathrm{CD} 4^{+}$cells to perform the study.

The presence of NKG2D ligands on the surface of HIV-1 p24 $\mathrm{Ag}^{+} \mathrm{HIV}$-infected cells, and the absence of these ligands on uninfected $\mathrm{CD}^{+}{ }^{+}$cells and the HIV-1 p24 $\mathrm{Ag}^{-}$compartment of the $\mathrm{HIV}$-infected cells, indicates that the production of HIV is necessary for the expression of NKG2D ligands. Expression of NKG2D ligand is dependent on activation of ATM/ATR, which is triggered by stress from the DNA damage pathway. ${ }^{34}$ HIV Vpr leads to activation of ATR. ${ }^{55-57}$ It is possible that Vpr is responsible for the expression of NKG2D ligand on infected cells.
If $\mathrm{Vpr}$ is indeed involved in NKG2D ligand expression, the induction of NKG2D ligands on the infected cells would seem counterproductive to the virus. However, Vpr has the advantage of inducing $\mathrm{G}_{2}$ arrest and increasing efficient preintegration events in the host cells and thus provides the virus an advantage in increasing the production of virions. ${ }^{55,56}$ The virus seems to offset the capability of activating NK cells efficiently by down-regulating the ligands for the 2B4 and NTB-A coreceptors on the infected cell surface. The decreased expression of CD48 and NTB-A is further compounded by our earlier observation that HLA-C and -E expression on infected cells may decrease the ability of some NKcell subsets ${ }^{20}$ to become activated despite NKG2D expression. Moreover, NK-cell receptors are modulated during the course of HIV infection. ${ }^{50,58,59}$ Future studies, using NK cells from viremic patients with HIV as effector cells to test cytotoxicity against infected targets will help to demonstrate the in vivo relevance of the modulation of activating ligands by the virus. This intricate interplay of surface receptor and ligand modulation seems to enable HIV to avoid the brunt of NK-cellmediated immune function.

\section{Acknowledgments}

We thank Professor A. Moretta from the University of Genoa, Genoa, Italy, for providing monoclonal antibodies, and Kelly Hudspeth for technical assistance.

This work was supported by National Institutes of Health grants AI16536, AI56923, and AI52809.

\section{Authorship}

Contribution: M.B., J.S. and J.G. performed research and analyzed data. M.F. performed research. D.M. designed research and wrote the manuscript. J.W. and E.B. designed and performed research, analyzed data, and wrote the manuscript. M.B. and J.S. contributed equally to this study.

Conflict-of-interest disclosure: The authors declare no competing financial interests.

Correspondence: Edward Barker, Department of Immunology and Microbiology, Rush University Medical Center, 1735 W. Harrison St., Chicago, IL 60612; e-mail: edward_barker@ rush.edu.

\section{References}

1. Bukowski JF, Woda BA, Habu S, Okumura K, Welsh RM. Natural killer cell depletion enhances virus synthesis and virus-induced hepatitis in vivo. J Immunol. 1983;131:1531-1538.

2. Biron CA, Byron KS, Sullivan JL. Severe herpesvirus infections in an adolescent without natural killer cells. N Engl J Med. 1989;320:1731-1735.

3. Lopez C, Kirkpatrick D, Read SE, et al. Correlation between low natural killing of fibroblasts infected with herpes simplex virus type 1 and susceptibility to herpesvirus infections. J Infect Dis. 1983;147:1030-1035.

4. Gazit R, Garty BZ, Monselise Y, et al. Expression of KIR2DL1 on the entire NK cell population: a possible novel immunodeficiency syndrome. Blood. 2004;103:1965-1966.

5. Lodoen MB, Lanier LL. Viral modulation of NK cell immunity. Nat Rev Microbiol. 2005;3:59-69.

6. Ciccone E, Pende D, Vitale M, et al. Self class I molecules protect normal cells from lysis mediated by autologous natural killer cells. Eur J Immunol. 1994;24:1003-1006.
7. Moretta A, Biassoni R, Bottino C, et al. Major histocompatibility complex class I-specific receptors on human natural killer and T lymphocytes. Immunol Rev. 1997;155:105-117.

8. Natarajan K, Dimasi N, Wang J, Mariuzza RA, Margulies $\mathrm{DH}$. Structure and function of natural killer cell receptors: multiple molecular solutions to self, nonself discrimination. Annu Rev Immunol. 2002;20:853-885.

9. Huard B, Fruh K. A role for MHC class I downregulation in NK cell lysis of herpes virus-infected cells. Eur J Immunol. 2000;30:509-515.

10. Long EO, Burshtyn DN, Clark WP, et al. Killer cell inhibitory receptors: diversity, specificity, and function. Immunol Rev. 1997;155:135-144.

11. Borrego F, Kabat J, Kim DK, et al. Structure and function of major histocompatibility complex $(\mathrm{MHC})$ class I specific receptors expressed on human natural killer (NK) cells. Mol Immunol. 2002;38:637-660.

12. Rojo S, Wagtmann N, Long EO. Binding of a soluble p70 killer cell inhibitory receptor to HLA-
$B \star 5101$ : requirement for all three p70 immunoglobulin domains. Eur J Immunol. 1997;27:568571.

13. Pende D, Biassoni R, Cantoni C, et al. The natural killer cell receptor specific for HLA-A allotypes: a novel member of the p58/p70 family of inhibitory receptors that is characterized by three immunoglobulin-like domains and is expressed as a 140-kD disulphide-linked dimer. J Exp Med. 1996; 184:505-518.

14. Braud VM, Allan DS, O'Callaghan CA, et al. HLA-E binds to natural killer cell receptors CD94/ NKG2A, B and C. Nature. 1998;391:795-799.

15. Brooks AG, Borrego F, Posch PE, et al. Specific recognition of HLA-E, but not classical, HLA class I molecules by soluble CD94/NKG2A and NK cells. J Immunol. 1999;162:305-313.

16. Colonna M, Navarro F, Bellon T, et al. A common inhibitory receptor for major histocompatibility complex class I molecules on human lymphoid and myelomonocytic cells. J Exp Med. 1997;186: 1809-1818. 
17. Lazetic S, Chang C, Houchins JP, Lanier LL, Phillips JH. Human natural killer cell receptors involved in $\mathrm{MHC}$ class I recognition are disulfidelinked heterodimers of CD94 and NKG2 subunits. J Immunol. 1996;157:4741-4745.

18. Burshtyn DN, Lam AS, Weston M, Gupta N, Warmerdam PA, Long EO. Conserved residues amino-terminal of cytoplasmic tyrosines contribute to the SHP-1-mediated inhibitory function of killer cell Ig-like receptors. J Immunol. 1999;162: 897-902.

19. Burshtyn DN, Scharenberg AM, Wagtmann N, et al. Recruitment of tyrosine phosphatase HCP by the killer cell inhibitor receptor. Immunity. 1996;4: 77-85.

20. Bonaparte MI, Barker E. Killing of human immunodeficiency virus-infected primary T-cell blasts by autologous natural killer cells is dependent on the ability of the virus to alter the expression of major histocompatibility complex class I molecules. Blood. 2004;104:2087-2094.

21. Bonaparte MI, Barker E. Inability of natural killer cells to destroy autologous HIV-infected T lymphocytes. Aids. 2003;17:487-494.

22. Cohen GB, Gandhi RT, Davis DM, et al. The selective downregulation of class I major histocom patibility complex proteins by HIV-1 protects HIVinfected cells from NK cells. Immunity. 1999;10: 661-671.

23. Uhrberg M, Valiante NM, Shum BP, et al. Human diversity in killer cell inhibitory receptor genes. Immunity. 1997;7:753-763.

24. Pietra G, Semino C, Cagnoni F, et al. Natural killer cells lyse autologous herpes simplex virus infected targets using cytolytic mechanisms distributed clonotypically. J Med Virol. 2000;62:354363.

25. Moretta A, Bottino C, Pende D, et al. Identification of four subsets of human CD3-CD16 + natural killer (NK) cells by the expression of clonally distributed functional surface molecules: correlation between subset assignment of NK clones and ability to mediate specific alloantigen recognition. J Exp Med. 1990;172:1589-1598.

26. Malnati MS, Lusso P, Ciccone E, Moretta A, Mor etta L, Long EO. Recognition of virus-infected cells by natural killer cell clones is controlled by polymorphic target cell elements. J Exp Med. 1993;178:961-969.

27. Lanier LL. NK cell recognition. Annu Rev Immunol. 2005;23:225-274.

28. Moretta A, Bottino C, Vitale M, et al. Activating receptors and coreceptors involved in human natural killer cell-mediated cytolysis. Annu Rev Immunol. 2001;19:197-223.

29. Augugliaro R, Parolini S, Castriconi R, et al. Selective cross-talk among natural cytotoxicity receptors in human natural killer cells. Eur J Immunol. 2003;33:1235-1241

30. Menier C, Riteau B, Carosella ED, Rouas-Freiss N. MICA triggering signal for NK cell tumor lysis is counteracted by HLA-G1-mediated inhibitory signal. Int J Cancer. 2002;100:63-70.

31. Wischhusen J, Friese MA, Mittelbronn M, Meyer- mann R, Weller M. HLA-E protects glioma cells from NKG2D-mediated immune responses in vitro: implications for immune escape in vivo. J Neuropathol Exp Neurol. 2005;64:523-528.

32. Pende D, Cantoni C, Rivera P, et al. Role of NKG2D in tumor cell lysis mediated by human NK cells: cooperation with natural cytotoxicity receptors and capability of recognizing tumors of nonepithelial origin. Eur J Immunol. 2001;31:10761086.

33. Mandelboim O, Lieberman N, Lev M, et al. Rec ognition of haemagglutinins on virus-infected cells by NKp46 activates lysis by human NK cells. Nature. 2001;409:1055-1060.

34. Gasser S, Orsulic S, Brown EJ, Raulet DH. The DNA damage pathway regulates innate immune system ligands of the NKG2D receptor. Nature. 2005;436:1186-1190.

35. Moretta L, Moretta A. Unravelling natural killer cell function: triggering and inhibitory human NK receptors. EMBO J. 2004;23:255-259.

36. Brown MH, Boles K, van der Merwe PA, Kumar V, Mathew PA, Barclay AN. 2B4, the natural killer and $T$ cell immunoglobulin superfamily surface protein, is a ligand for CD48. J Exp Med. 1998; 188:2083-2090.

37. Flaig RM, Stark S, Watzl C. NTB-A activates NK cells via homophilic interaction. J Immunol. 2004; 172:6524-6527.

38. Falco M, Marcenaro E, Romeo E, et al. Ho mophilic interaction of NTBA, a member of the CD2 molecular family: induction of cytotoxicity and cytokine release in human NK cells. Eur J Immunol. 2004;34:1663-1672.

39. Pende D, Spaggiari GM, Marcenaro S, et al. Analysis of the receptor-ligand interactions in the natural killer-mediated lysis of freshly isolated myeloid or lymphoblastic leukemias: evidence for the involvement of the Poliovirus receptor (CD155) and Nectin-2 (CD112). Blood. 2005;105: 2066-2073.

40. O'Doherty U, Swiggard WJ, Malim MH. Human immunodeficiency virus type 1 spinoculation enhances infection through virus binding. J Virol. 2000;74:10074-10080.

41. Gorny MK, Gianakakos V, Sharpe S, ZollaPazner S. Generation of human monoclonal antibodies to human immunodeficiency virus. Proc Natl Acad Sci U S A. 1989;86:1624-1628.

42. Simm M, Shahabuddin M, Chao W, Allan JS, Volsky DJ. Aberrant Gag protein composition of a human immunodeficiency virus type 1 vif mutant produced in primary lymphocytes. J Virol. 1995; 69:4582-4586

43. Pende D, Parolini S, Pessino A, et al. Identification and molecular characterization of NKp30, a novel triggering receptor involved in natural cytotoxicity mediated by human natural killer cells. J Exp Med. 1999;190:1505-1516.

44. Sivori S, Vitale M, Morelli L, et al. p46, a novel natural killer cell-specific surface molecule that mediates cell activation. J Exp Med. 1997;186: 1129-1136.

45. Arnon TI, Lev M, Katz G, Chernobrov Y, Porgador
A, Mandelboim O. Recognition of viral hemagglutinins by NKp44 but not by NKp30. Eur J Immunol. 2001;31:2680-2689.

46. Vieillard V, Strominger JL, Debre P. NK cytotoxicity against CD4+ T cells during HIV-1 infection: A gp41 peptide induces the expression of an NKp44 ligand. Proc Natl Acad Sci U S A. 2005; 102:10981-10986.

47. Bauer S, Groh V, Wu J, et al. Activation of NK cells and T cells by NKG2D, a receptor for stressinducible MICA. Science. 1999;285:727-729.

48. Vivier E, Tomasello E, Paul P. Lymphocyte activation via NKG2D: towards a new paradigm in immune recognition. Curr Opin Immunol. 2002;14: 306-311.

49. Pende D, Rivera P, Marcenaro S, et al. Major histocompatibility complex class I-related chain A and UL16-binding protein expression on tumor cell lines of different histotypes: analysis of tumor susceptibility to NKG2D-dependent natural killer cell cytotoxicity. Cancer Res. 2002;62:6178-6186.

50. Mavilio D, Benjamin J, Daucher M, et al. Natural killer cells in HIV-1 infection: dichotomous effects of viremia on inhibitory and activating receptors and their functional correlates. Proc Natl Acad Sci U S A. 2003;100:15011-15016.

51. Vitale M, Bottino C, Sivori S, et al. NKp44, a novel triggering surface molecule specifically expressed by activated natural killer cells, is involved in non-major histocompatibility complexrestricted tumor cell lysis. J Exp Med. 1998;187: 2065-2072.

52. Cerboni C, Neri F, Casartelli N, et al. Human immunodeficiency virus-1 nef protein down modulates the ligands of the activating receptor NKG2D and inhibits natural killer cell-mediated cytotoxicity. J Gen Virol. 2007;88:242-250.

53. Molinero LL, Fuertes MB, Rabinovich GA, Fainboim L, Zwirner NW. Activation-induced expression of MICA on T lymphocytes involves engage ment of CD3 and CD28. J Leukoc Biol. 2002;71: 791-797.

54. Bryceson $\mathrm{YT}$, March ME, Ljunggren HG, Long EO. Synergy among receptors on resting NK cells for the activation of natural cytotoxicity and cytokine secretion. Blood. 2006;107:159-166.

55. Andersen JL, Planelles V. The role of Vpr in HIV-1 pathogenesis. Curr HIV Res. 2005;3:43-51.

56. Zimmerman ES, Chen J, Andersen JL, et al. Human immunodeficiency virus type $1 \mathrm{Vpr}$-mediated G2 arrest requires Rad17 and Hus1 and induces nuclear BRCA1 and gamma-H2AX focus formation. Mol Cell Biol. 2004;24:9286-9294.

57. Roshal M, Kim B, Zhu Y, Nghiem P, Planelles V. Activation of the ATR-mediated DNA damage response by the HIV-1 viral protein R. J Biol Chem. 2003;278:25879-25886.

58. Mavilio D, Lombardo G, Benjamin J, et al. Characterization of CD56-/CD16 + natural killer (NK) cells: a highly dysfunctional NK subset expanded in HIV-infected viremic individuals. Proc Nat Acad Sci U S A. 2005;102:2886-2891.

59. Fauci AS, Mavilio D, Kottilil S. NK cells in HIV infection: paradigm for protection or targets for ambush. Nat Rev Immunol. 2005:5:835-843. 